\title{
Neurophysiological monitoring of displaced odontoid fracture reduction in a 3-year-old male
}

\author{
Shandy Fox $\mathbb{B}^{1} \cdot$ Lauren Allen $^{1} \cdot$ Jonathan Norton ${ }^{1}$
}

Received: 10 February 2018 / Revised: 25 April 2018 / Accepted: 7 May 2018

(c) International Spinal Cord Society 2018

\begin{abstract}
Introduction Odontoid fractures in young children are rare. Most authors advocate for closed reduction and external stabilization as first line treatment. Unlike adults, young children are much less amenable to an awake reduction for real-time assessment of neurological function. We used spinal cord monitoring, as used in spine surgery, to assess the function of the spinal cord during the closed reduction in our 31-month-old patient.

Case presentation A 31-month-old male presented with a displaced odontoid fracture and ASIA C spinal cord injury. Given his age, closed reduction and halo application were completed under general anesthesia guided by neuromonitoring. A lessthan-ideal reduction initially was accepted due to a decline in motor-evoked potentials. Subsequently, there was no change in neurological status. The reduction was repeated under anesthesia, with monitoring, a number of times until good correction was achieved. Ultimately, a surgical fusion was required due to ligamentous instability. The child achieved a very good neurological outcome and a stable spine.

Discussion Neuromonitoring is an important adjunct to closed reductions when complete and reliable neurological assessment is not possible.
\end{abstract}

\section{Introduction}

Pediatric spine injuries are relatively rare with incidence in the literature ranging from 0.2 to $0.5 \%$ of all fractures and 2.7 to $9 \%$ of all spinal injuries [1,2]. Younger patients are more likely to present with upper cervical spine involvement due to their anatomy; a greater head-toneck ratio, ligamentous laxity, underdeveloped neck muscles, horizontal facets, and physiologic wedging of the vertebra [3-6]. Furthermore, C2 is particularly susceptible given that the cartilaginous plate between the odontoid process and the axis does not fuse until the age of 5-7 [6-8].

Given the rarity of these injuries, there is little guidance in the definitive management of each injury pattern. Most

Jonathan Norton

j.norton@usask.ca

1 Department of Surgery, University of Saskatchewan Royal University Hospital, 103 Hospital Drive, Saskatoon, SK S7N 0W6, Canada authors continue to support closed reduction, external stabilization, and close follow-up [6, 9-13]. The halo vest is still the most widely used with an excellent fusion rate reported from 80 to $93 \%$ and immobilization ranging from 10 to 18 weeks [9-11].

Mandaback et al. advocate the application of the halo device under ketamine procedural sedation with any reduction done under fluoroscopic guidance [14]. Although procedural sedation is necessary for both analgesia and ease of reduction in the young, reliable neurological monitoring is no longer possible. The adult literature advocates for an awake spinal reduction to monitor neurological deterioration.

Neurophysiological monitoring has been increasingly incorporated into the operating room for spinal interventions to assess the patient's baseline status and intraoperative changes [15]. This technique could be incorporated into procedures such as spinal closed reduction in young patients who are otherwise unable to cooperate with an awake closed reduction.

We present this case to highlight the role of neurophysiological monitoring in the closed reduction of a displaced odontoid fracture in a 3-year-old male. 


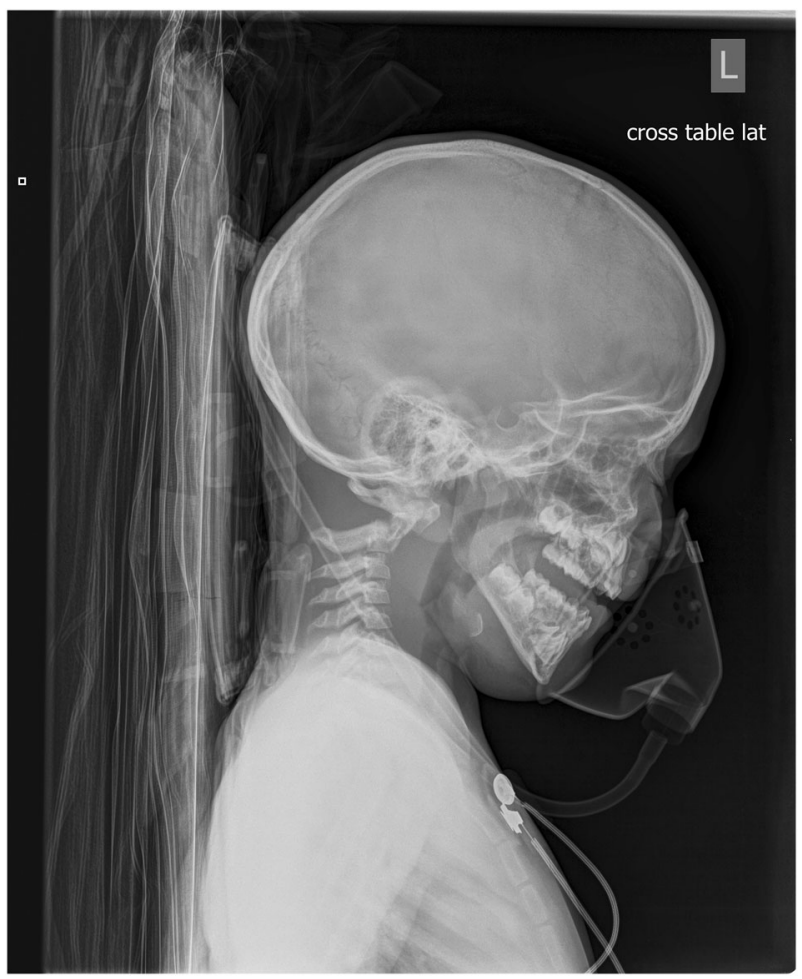

Fig. 1 Initial spine board X-ray taken in the peripheral hospital showing significant odontoid displacement and the inappropriate head position

\section{Case presentation}

A 31-month-old male presented to local hospital after being ejected off of an all terain vehicle (ATV). He was not wearing a helmet. Level of consciousness was documented as decreased at the site. He was placed on a spine board and transferred to a local hospital, where the ATLS protocol was initiated and initial imaging was obtained.

Plain radiograph of the lateral cervical spine (Fig. 1) shows inappropriate positioning of the child on the spine board. There is abnormal flexion alignment and evidence of an odontoid fracture. Excessive flexion has accentuated the displacement of the fracture site. This was promptly identified and addressed by the local medical team.

CT scan confirmed a flexion-type odontoid fracture with comminution and extension into the $\mathrm{C} 2$ body (Fig. 2). There was anterior displacement of the odontoid as well as the ring of $\mathrm{C} 1$. A left midshaft clavicle fracture and nondisplaced mandibular fractures were also noted.

The patient was placed in a cervical orthosis with a towel rolled between his shoulder blades. He was medically stabilized and transferred via air transport to the regional trauma center. His medical and surgical history revealed a dog bite one year prior requiring skin grafting to the right

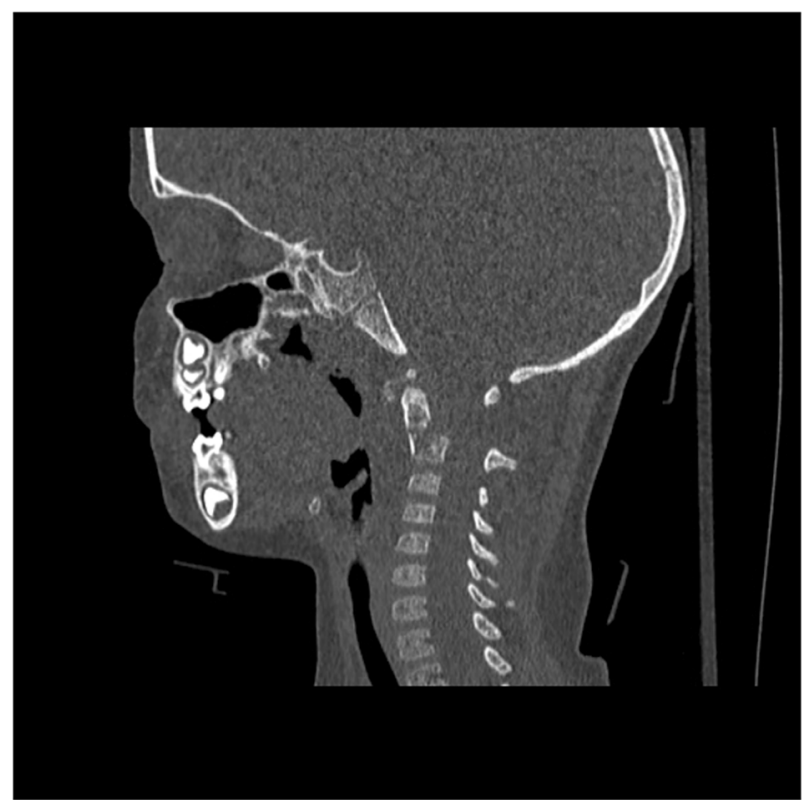

Fig. 2 Plain CT scan of the C-spine demonstrating the injury

side of his face. He was otherwise healthy and immunizations were up to date.

Upon arrival his GCS was 12. He was moving all limbs, however, a detailed neurological exam was difficult due to his age, pain, and distress. Although we could not accurately grade his strength, movement was noted in all myotomes from C5-T1 and L2-S1 and appeared to be antigravity or less. Sensation was grossly intact. Reflexes were symmetrical in both upper and lower extremities. Plantars were downgoing. Tone was normal. Clinical exam was most consistent with an ASIA C spinal cord injury. Plain radiographs were repeated that showed ongoing anterior displacement of the $\mathrm{C} 2$ fracture (Fig. 3).

The patient required general anesthesia to undergo MRI of his brain and spinal cord. The MRI showed significant soft tissue disruption posterior around the ring of $\mathrm{C} 1 / 2$ as well as cord edema extending from $\mathrm{C} 1$ to $\mathrm{C} 4$ (Fig. 4). $\mathrm{He}$ was taken to the operating room (OR) immediately following the MRI, under the same anesthesia, for closed reduction of the odontoid. Motor-evoked potentials (MEPs) and somatosensory-evoked potentials were elicited using standard protocols [15]. There was evidence of spinal cord dysfunction, with delayed, polyphasic potentials in all extremities [16]. These were most delayed in the right arm.

A small halo Bremer ring and eight pins were placed followed by fitting of the vest. A gentle and controlled reduction was carried out by applying manual traction and extension. This was guided by fluoroscopy and neurophysiological monitoring. Once alignment was achieved, the halo and vest were secured. The patient was then propped upright to further assess position and settling of the fracture 


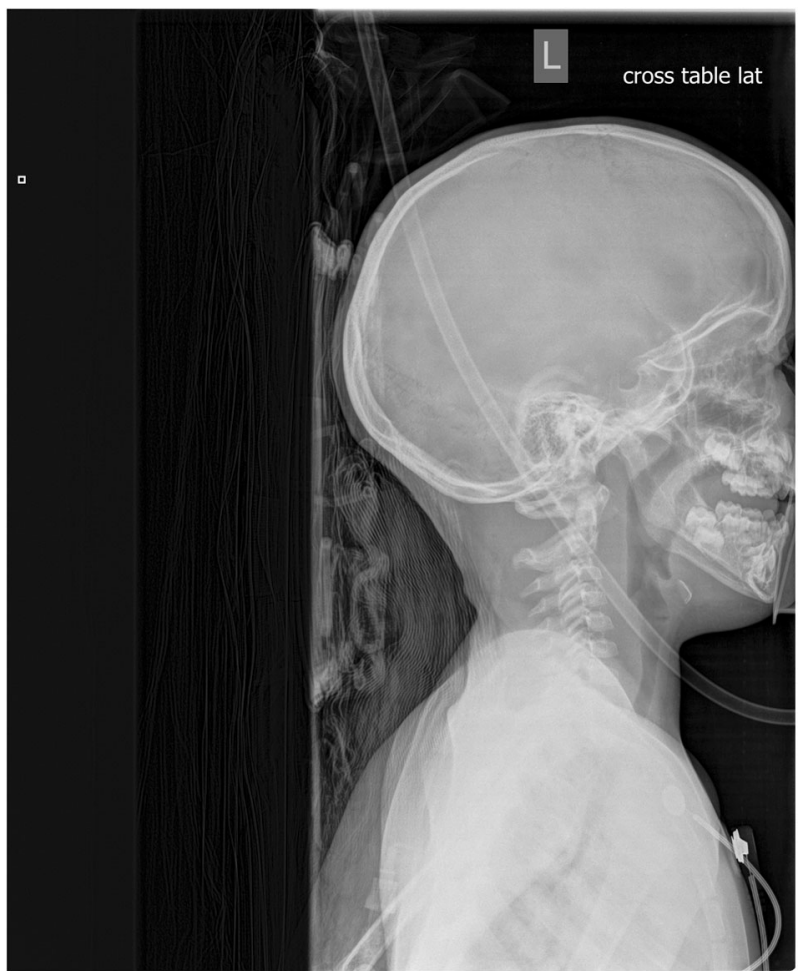

Fig. 3 Following re-positioning on the spine board there is a reduction in the displacement and a more neutral position

in sitting. Some translation was noted and repeat reduction was completed with the halo ring and vest. Final radiographs showed acceptable reduction and restoration of the occipitocervical alignment (Fig. 5). Clinically, however, his neck was in an extended position. Further flexion was attempted, but resulted in a significant decrease in the MEPs (>80\%) which returned to slightly larger than baseline in the extended position. This extended neck position, with adequate fracture reduction and MEPs, was accepted, with plans to gradually increase flexion as the fracture stabilized over time.

The patient's clavicle and mandible fractures were treated non-operatively. Following the closed reduction, he remained intubated in PICU with significant airway edema. He required tube feeds through most of his stay secondary to his mandible fracture and extended neck position.

Initially his right arm was quite weak. Physical and occupational therapy began rehabilitation and he progressed well, mobilizing independently and using all of his limbs for play by the time he was discharged 8.5 weeks later.

Seven weeks following his injury he was taken to the OR for adjustment of his alignment. Under general anesthesia, fluoroscopy and neuromonitoring, he was placed in a more neutral position. Again, a mild decrease of the MEPs $(\sim 35 \%)$ was noted with further flexion and this was thus abandoned. Despite this, he was in a more neutral position

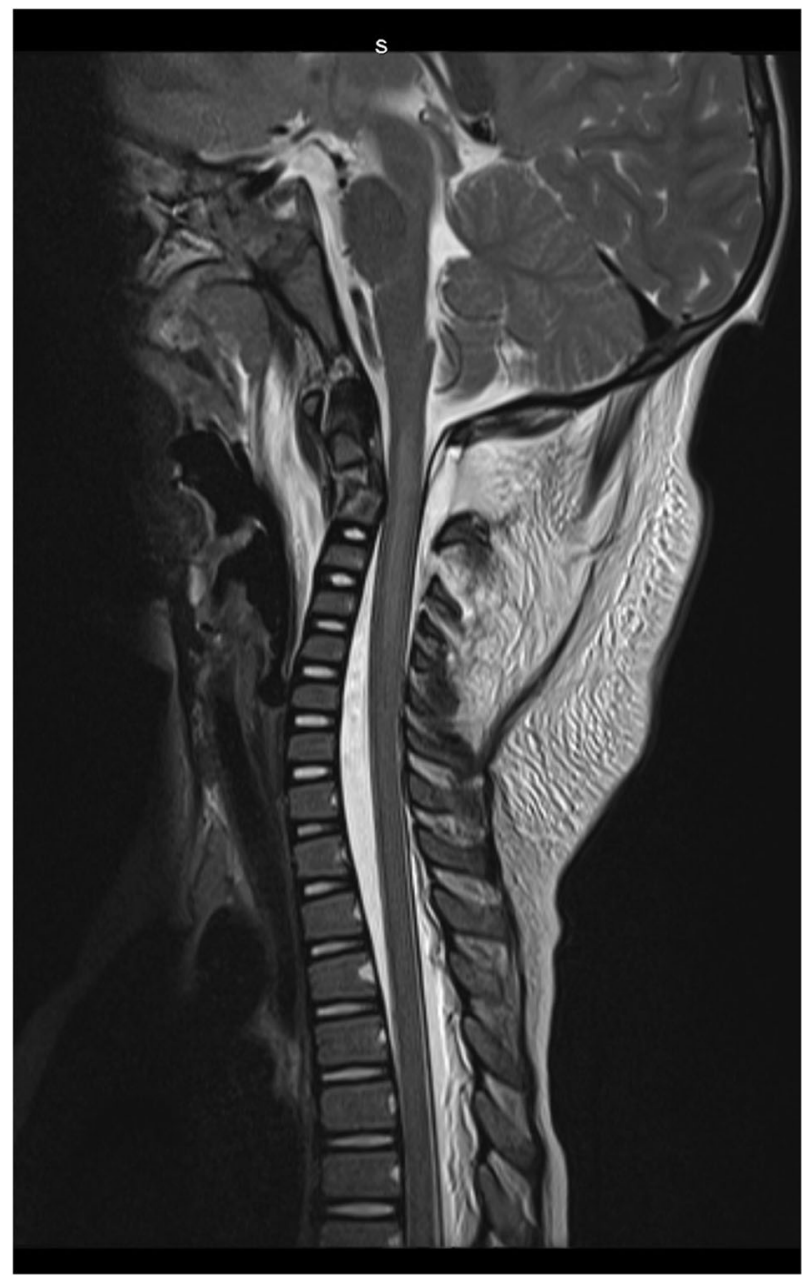

Fig. 4 Prior to reduction of the injury using the halo, the MRI of the spinal cord shows a multi-level region of signal change

than previous and he was kept in the halo vest to promote further healing of his ligamentous injury.

The patient was brought back to the OR 13 weeks following his injury. Following general anesthesia, neurophysiological assessment was repeated. Evaluation of alignment and stability was assessed with gentle controlled motion with neuromonitoring and fluoroscopic guidance. Mild kyphosis was identified at $\mathrm{C} 2 / 3$ with no evidence of instability on maximal flexion or extension. He was placed in a Miami $\mathbf{J}$ cervical collar for four weeks.

At 6 months post injury, he showed progressive kyphosis at $\mathrm{C} 2 / 3$ and $\mathrm{C} 3 / 4$. Posterior instrumentation and fusion of $\mathrm{C} 2-\mathrm{C} 4$ were performed using pars screws at $\mathrm{C} 2$, lateral mass screws at $\mathrm{C} 3$ and $\mathrm{C} 4$, and iliac autograft. This was followed by 8 weeks of halo vest immobilization and collar for another month. At one year post injury, he was participating in most recreational activities. Examination revealed only a mildly increased tone through the right leg and difficulty with single-legged hops. 


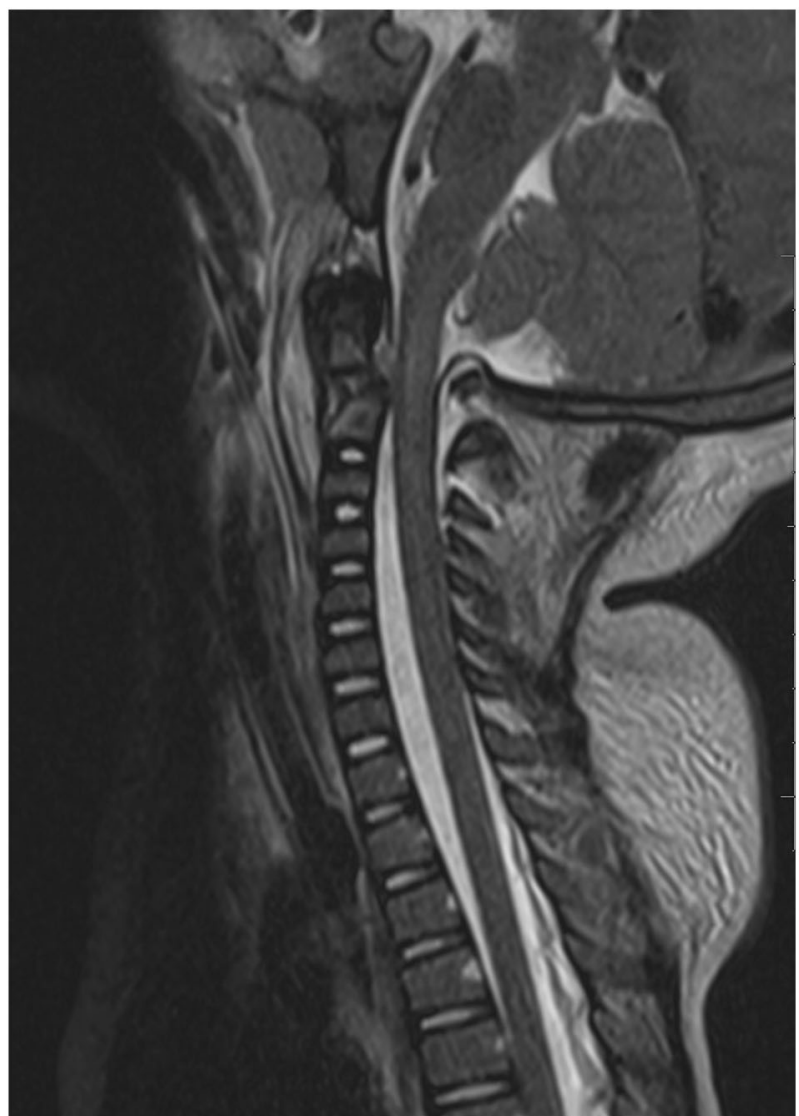

Fig. 5 After closed reduction, and with the halo applied, the signal change within the spinal cord is reduced in size and there is cerebrospinal fluid (CSF) space in front of the spinal cord despite a persistent deformity

\section{Discussion}

Fortunately, upper cervical spine injuries in children are rare, but are over represented due to their head and neck anatomy. As illustrated in this case, careful positioning of the injured child on a spine board is imperative to avoid any worsening of a possible cervical spine injury [17, 18]. A child's large head size necessitates elevation of the torso on the spine board in order to avoid undue neck flexion.

Neurological deficits associated with cervical spine injuries in children have been reported as high as 52-55\% $[1,2]$. The outcomes following spinal cord injury in young children are much better than in adults [19]. The initial neurological status seems to represent an important factor for prognosis and clinical outcome, with incomplete injuries being most favorable [1]. As discussed above, our patient also demonstrated good recovery after presenting with an incomplete injury.

The paucity of evidence in managing these injuries has led to a variety of treatment approaches. Ozkan et al. highlighted six cases of odontoid fracture in children, five type II and one type III, that did well with application of a
Philadelphia collar for 3-5 weeks [1]. Many other authors advocate for halo fixation. Osenback and Menezes found that the halo vest provided superior immobilization in cervical spine injuries and minimal morbidity in patients as young as 1 year of age $[20,21]$. In contrary to these methods, some recent literature supports early surgical treatment [1]. Indications for surgical intervention include unstable injuries with progressive neurological deficit, fracture dislocations, or progressive deformity with compression of neurological structures [22]. Ozkan et al. performed surgical fixation in $46.7 \%$ of their 75 patients with no reports of growth arrest, spinal deformities, neurologic decline, or revision [2].

With such paucity and variability in the literature on this topic, it is unknown whether operative intervention aids fracture union and functional outcome in small children. In this case, the team elected to manage the fracture with closed reduction and halo vest. The optimal method of performing a closed reduction in an adult is to reduce the fracture while the patient is awake to monitor neurological status. Many patients are not able to participate in this type of reduction, either because of age, cognitive ability, or concurrent injuries. Although performing the reduction in an MRI scanner is an option, only the position of the spinal cord can be monitored, not function [23]. As an alternative, our team used evoked potentials during reduction to give some measure of spinal cord function [24].

In this case, evoked potentials were able to guide and modify fracture reduction and neck positioning. We believe that the use of the evoked potentials prevented a significant worsening of neurological function. Although the fracture went on to bony union, surgical stabilization was eventually required for his associated ligamentous injury.

Acknowledgements We thank the patient's family for their permission to describe this case. We are grateful to Dr. Martin Stockwell (Anaesthesia, Royal University Hospital), for excellent anesthetic care during all procedures.

\section{Compliance with ethical standards}

Conflict of interest The authors declare that they have no conflict of interest.

\section{References}

1. Ozkan N, Wrede K, Ardeshiri A, Sariaslan Z, Stein KP, Dammann $\mathrm{P}$, et al. Management of traumatic spinal injuries in children and young adults. Child's Nerv Syst. 2015;31:1139-1148.

2. Duhem R, Tonnelle V, Vinchon M, Assaker R. Unstable upper pediatric cervical spine injuries: report of 28 cases and review of the literature. Child's Nerv Syst. 2008;24:343-348.

3. Rusin JA, Ruess L, Daulton RS. New C2 synchondrosal fracture classification system. Pediatr Radiol. 2015;45:872-881. 
4. Lustrin ES, Karakas SP, Ortiz AO. Pediatric cervical spine: normal anatomy, variants and trauma. Radiographics. 2003;23:539-560.

5. O'Brien WT, Shen P, Lee P. The dens: normal development, developmental variants and anomalies, and traumatic injuries. J Clin Imaging Sci. 2015;5:38.

6. Fulkerston DH, Hwang SW, Patel AJ, Jea A. Open reduction and internal fixation for angulated, unstable odontoid synchondrosis fracture in children: a safe alternative to halo fixation. J Neurosurg Pediatr. 2012;9:35-41.

7. Ogden JA. Radiology of postnatal skeletal development. XII. The second cervical vertebra. Skelet Radiol. 1984;12:169-177.

8. Wong S, Zovickian J, Hou Y, Sonne C, Pang D. Traumatic rupture of the neurocentral synchondrosis of the axis in a child. $\mathrm{J}$ Neurosurg Pediatr. 2014;13:548-552.

9. Fassett DR, McCall T, Brockmeyer DL. Odontoid synchondrosis fractures in children. Neurosurg Focus. 2006;20:E7.

10. Kinkpe CV, Dansokho AV, Coulibaly NF, Niane MM, Seye SI, Sales DGJ. Fracture of the odontoid process in children: a case report. Orthop Traumatol Surg Res. 2009;95:234-236.

11. Odent T, Langlais J, Glorion C, Kassis B, Bataille J, Pouliquen JC. Fractures of the odontoid process: a report of 15 cases in children younger than 6 years. J Pediatr Orthop. 1999;19:51-54.

12. Schippers N, Konings P, Hassler W, Sommer B. Typical and atypical fractures of the odontoid process in young children. Report of two cases and a review of the literature. Acta Neurochir (Wien). 1996;138:524-530.

13. Mueller OM, Gasser T, Hellwig A, Dohna-Schwake C, Sure U. Unstable cervical spine injury in a toddler: technical note. Child's Nerv Syst. 2010;26:1625-1631.

14. Mandabach M, Ruge JR, Hahn YS, McLone DG. Pediatric axis fractures: early halo immobilization, management and outcome. Pediatr Neurosurg. 1993;19:225-32.
15. Nuwer MR, Emerson RG, Galloway GM, Legatt AD, Lopez J, Minahan RE, et al. Evidence-based guideline update: intraoperative spinal monitoring with somatosensory and transcranial electrical motor evoked potentials: report of the Therapeutics and Technology Assessment Subcommittee of the American Academy of Neurology and the American Clinical Neurophysiology Society. Neurology. 2012;78:585-589.

16. Calancie B, Molano MR. Alarm criteria for motor-evoked potentials: what's wrong with the "presence-or-abscence" approach? Spine. 2008;33:406-414.

17. Easter JS, Barkin R, Rosen CL, Ban K. Cervical spine injuries in children, part II: management and special considerations. J Emerg Med. 2011;41:252-256.

18. Nypaver M, Treloar D. Neutral cervical spine positioning in children. Ann Emerg Med. 1994;23:208-211.

19. Greenberg JS, Ruutiainen AT, Kim H. Rehabilitation of pediatric spinal cord injury: from acute medical care to rehabilitation and beyond. J Pediatr Rehabil Med. 2009;2:13-27.

20. Osenback RK, Menezes AH. Pediatric spinal cord and vertebral column injury. Neurosurgery. 1992;30:385-390.

21. Basu S. Spinal injuries in children. Front Neurol. 2012;3:96.

22. Viccellio P, Simon H, Pressman BD, Shah MN, Mower WR, Hoffman JR. A prospective multicenter study of cervical spine injury in children. Pediatrics. 2001;108:E20.

23. Darsaut TE, Sartawi MM, Dhaliwal P, Fox RJ. Rapid magnetic resonance imaging-guided reduction of cranioverterbal junction deformities. J Neurosurg Spine. 2009;10:27-32.

24. Di Lazzaro V, Oliviero A, Profice P, Ferrara L, Saturno E, Pilato $\mathrm{F}$, et al. The diagnostic value of motor evoked potentials. Clin Neurophysiol. 1999;110:1297-1307. 